\title{
High-Grade Extremity Myxofibrosarcoma with Synchronous Mesenteric Leiomyosarcoma: Double Primary Sarcomas
}

\author{
Milljae Shin, Sung-Joo Kim \\ Department of Surgery, Samsung Medical Center, Sungkyunkwan University School of Medicine, Seoul, Korea \\ Email: milljae.shin@gmail.com
}

Received May 30, 2013; revised July 1, 2013; accepted July 9, 2013

Copyright (C) 2013 Milljae Shin, Sung-Joo Kim. This is an open access article distributed under the Creative Commons Attribution License, which permits unrestricted use, distribution, and reproduction in any medium, provided the original work is properly cited.

\begin{abstract}
Here, we report the case of a 47-year-old male who presented with a painless palpable mass in the right shoulder. This extremity tumor was diagnosed as a high-grade myxofibrosarcoma after a wide excision. Simultaneously, a synchronous mesenteric mass was discovered, which proved to be a leiomyosarcoma.
\end{abstract}

Keywords: Double Primary; Leiomyosarcoma; Myxofibrosarcoma; Synchronous Sarcoma

\section{Introduction}

Soft tissue sarcomas are a heterogeneous group of malignancies that arise from primitive mesenchymal cells [1]. Soft tissue sarcomas may be associated with the development of other malignancies in several genetic conditions including neurofibromatosis [2], familial adenomatous polyposis [2], retinoblastoma [3], and Li-Fraumeni syndrome $[4,5]$. Aside from patients with family histories, it is extremely rare to see two distinct sarcomas of different histological types simultaneously and sporadically presenting in one individual.

Here, we report a case in which a mesenteric leiomyosarcoma (LMS) was discovered during treatment for an extremity myxofibrosarcoma (MFS). We speculate on the possibility of a concurrent LMS mimicking the metastasis of a primary high-grade MFS. To our knowledge, this is the first published case report in the English literature of a patient with synchronous development of a mesenteric LMS and an extremity MFS.

\section{Case History}

A 47-year-old man presented with a palpable mass in the right shoulder, which had been present for two months and had doubled in size during that time. He had undergone endoscopic mucosal resection for early gastric cancer six years before. He had no other significant medical history and his family history was unremarkable. He had experienced an obvious weight loss $(3 \mathrm{~kg} / 1$ month, BMI
$24.5 \mathrm{~kg} / \mathrm{m}^{2}$ ). During the physical examination, we found a palpable mobile mass that was firm, painless, and non-tender to the touch. He was otherwise well. All blood laboratory tests were within normal limits.

Since the clinical features were diagnostic of extremity soft tissue sarcoma, a wide excision of the pathologic lesion was performed in August 2011. Visible and palpable tissue was resected in an en bloc fashion. All involved muscle bundles and overlying skin were included in the resection. The tumor measured about $6 \times 4.5 \times 3$ $\mathrm{cm}$ and did not seem to have an infiltrative growth pattern. The lesion was identified as a high-grade MFS which was categorized as $3 / 3$ according to the French Federation of Cancer Centers Sarcoma Group (FNCLCC) grading system. The sample showed conspicuous solid and hypercellular components with high pleomorphism, numerous atypical mitotic figures (20 per 10 high-power field), and confluent areas of necrosis in over half of the sample (Figure 1). The surrounding skeletal muscle and skin were not involved. According to the immunohistochemistry report, tumor cells were positive for $\mathrm{p} 53$ and vimentin but negative for cytokeratin (CK), S-100 protein, CD68 and alpha-smooth muscle actin (SMA). The $\mathrm{Ki}-67$ index was $50 \%$.

To investigate the high-grade soft tissue extremity sarcoma, whole-body ${ }^{18} \mathrm{~F}$-2-fluoro-2-deoxy-D-glucose $\left({ }^{18} \mathrm{FDG}\right)$ positron emission tomography (PET) scan was performed to determine the patient's stage. The scan showed a hypermetabolic mass $\left(\mathrm{SUV}_{\max }=15.1\right)$ in the 


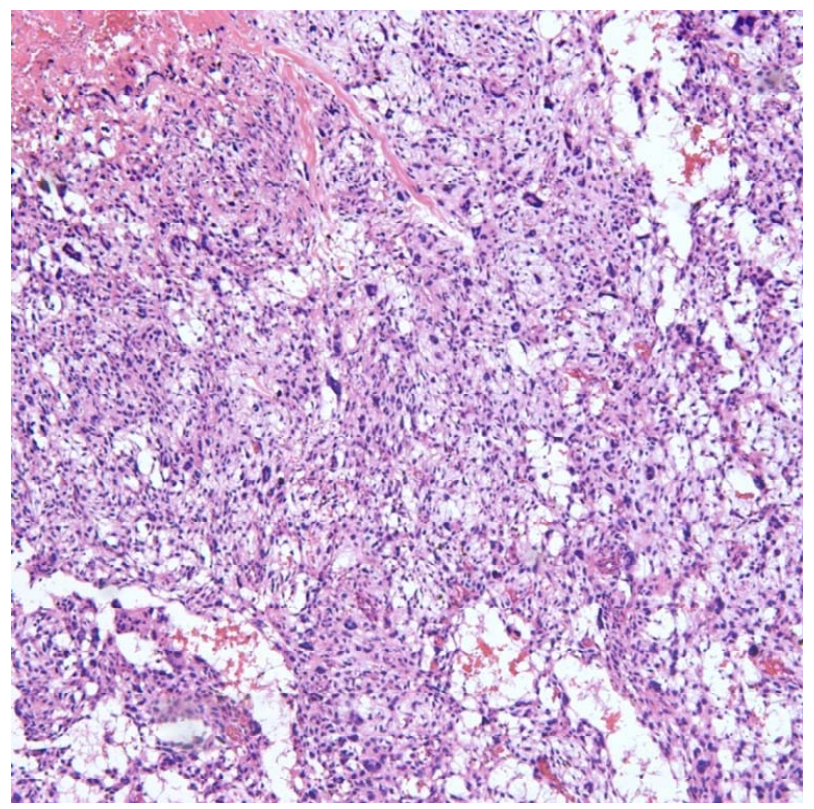

Figure 1. Microscopic view of the high-grade extremity myxofibrosarcoma (hematoxylin-eosin staining; magnification $\times 100)$.

right lower quadrant of abdomen, which was suspicious for malignancy (Figure 2(a)). There was no evidence of increased FDG uptake in the right shoulder area or in both lungs. A second CT scan of the abdomen revealed the presence of a well-demarcated solid sized $4.7 \times 5.0$ $\mathrm{cm}$ and a cystic mass in mesentery of the right lower quadrant (Figure 2(b)). At this point, metastasis was suspected but no diagnosis was made. Because MFS is known for its chemo-sensitivity, we decided to proceed with the upfront palliative chemotherapy, and if the tumor remained stable, would follow with a surgical metastatectomy for the intra-abdominal mass.

From October 2011 to January 2012, the patient received four cycles of chemotherapy. His regimen included $50 \mathrm{mg} / \mathrm{m}^{2} / 4$ days of doxorubicin in conjunction with two-hour infusions of $2.0 \mathrm{~g} / \mathrm{m}^{2} /$ day ifosfamide $[6,7]$. Mesna equimolar to the ifosfamide was administered immediately before and four hours after the ifosfamide. Each cycle of chemotherapy was followed by G-CSF prophylaxis. Radiologic images of the metastatic lesion, both ${ }^{18}$ FDG-PET and abdominal CT scans, were taken after the final cycle. The malignant mass in the right lower abdomen had no significant changes in size $(5.2 \times 5.0$ $\mathrm{cm})$ or FDG uptake $\left(\mathrm{SUV}_{\max }=15.3\right)$ from what was previously noted. There was no evidence of any abnormal FDG uptake or a definite new lesion, which would have suggested metastasis. After these four cycles of chemotherapy, the patient's performance status was 1 (symptomatic; fully ambulatory) and he was judged to have stable disease. The patient was scheduled to complete chemotherapy after surgical resection.

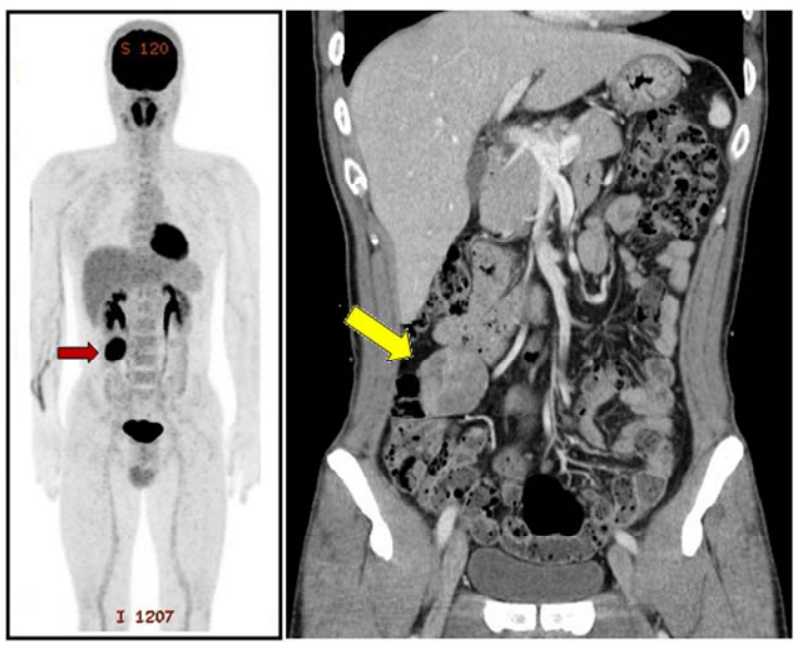

(a)

(b)

Figure 2. Images of intra-abdominal mass discovered: (a) Whole-body ${ }^{18}$ F-2-fluoro-2-deoxy-D-glucose positron emission tomography, red arrow; (b) Abdominal computed tomography, yellow arrow.

The mesenteric mass on the right lower quadrant of the abdomen was treated by surgical excision followed by an incidental appendectomy at the department of surgery in February 2012. During the laparotomy, we identified a round encapsulated mass measuring $5.3 \times 4.5 \times 4.0 \mathrm{~cm}$ rising from the mesentery of the terminal ileum. There was no evidence of invasion into the small intestine or adjacent tissues. The mass was removed via a simple excision. The cut surface of this smooth mass was rubbery, pale yellow in color, myxoid, and nodular without evidence of hemorrhagic necrosis (Figure 3).

Histopathologic examination of the mass revealed an undifferentiated pleomorphic sarcoma (Figure 4(a)). Besides high cellularity, it was composed of highly pleomorphic and spindle-shaped cells arranged in an interlacing fascicular pattern, showing bizarre nuclei and increased mitotic figures (10 per 10 high-power field). The surgical margins were tumor-free. Immunohistochemical samples stained with alpha-smooth muscle actin (SMA) and desmin were diffuse and strongly positive (Figures 4(b) and (c)). The tumor cells demonstrated a negative reaction for S-100 protein, CD68, c-MET and nestin. The $\mathrm{Ki}-67$ index was $35 \%$. The results are summarized in Table 1. Accordingly, immunohistochemistry of the mesenteric mass established the diagnosis of LMS, which was categorized as $3 / 3$ according to the FNCLCC grading system.

The patient had an unremarkable recovery and was discharged within one week. The patient subsequently received adjuvant radiation therapy four weeks after surgery in March 2012. He was tolerable at the time of the submission of this article. 


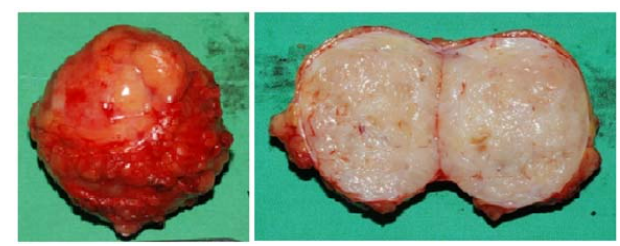

Figure 3. Bulk of the sarcoma manifested by a mesenteric mass.

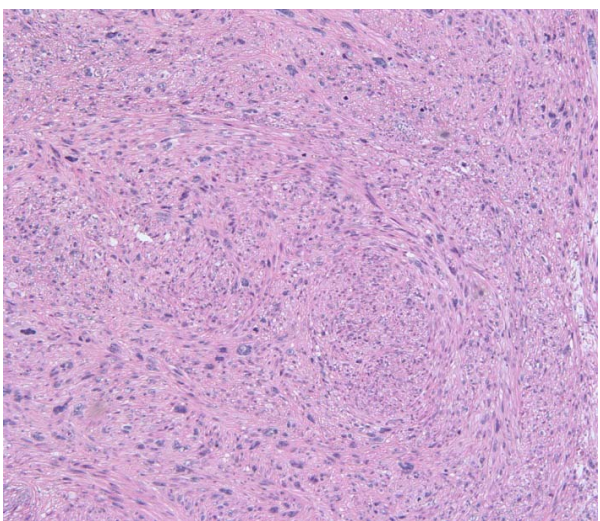

(a)

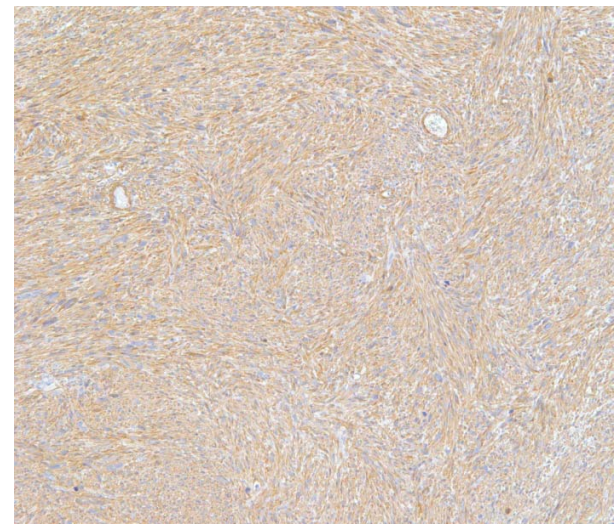

(b)

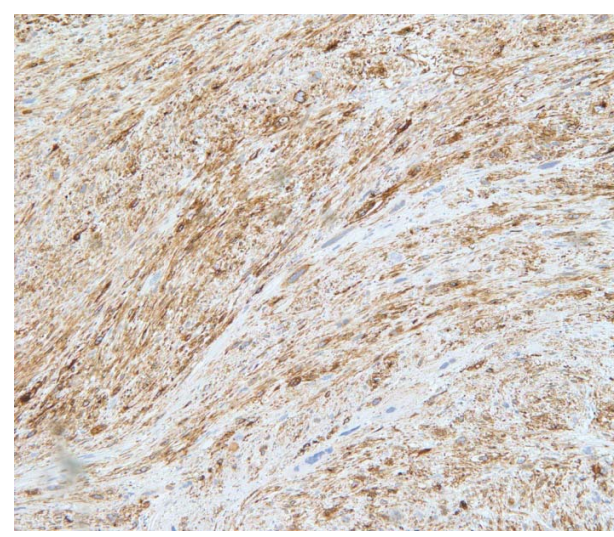

(c)

Figure 4. Microscopic view of the mesenteric mass (hematoxylin-eosin staining and immunohistochemical staining; magnification $\times 100$ ): Histological section (a) and immunereactivity for SMA (b) and desmin (c).
Table 1. Immunohistochemistry results between extremity mass and mesenteric mass.

\begin{tabular}{ccc}
\hline Antigen & Extremity mass & Mesenteric mass \\
\hline p53 & + & ND \\
Vimentin & + & ND \\
CK (cytokeratin) & - & ND \\
S-100 protein & - & - \\
SMA (a-smooth muscle actin) & - & + \\
CD68 & - & - \\
Desmin & ND & + \\
c-MET & ND & - \\
Nestin & ND & - \\
Ki-67 & $+(50 \%)$ & $+(35 \%)$ \\
Final confirmed diagnosis & Myxofibrosarcoma & Leiomyosarcoma \\
\hline , negative; + positive; ND, not done. & &
\end{tabular}

\section{Discussion}

MFS is one of the most common sarcomas in the extremities of elderly patients [8]. It arises more frequently in subcutaneous tissues than in deep soft tissues [9]. Distinctive histological features vary from a hypocellular, mainly myxoid, and purely spindle-cell appearance (lowgrade neoplasm) to high-grade, pleomorphic (malignant fibrous histiocytoma-like) lesions with multinucleated giant cells, high mitotic activity, and areas of necrosis [9]. Because of morphological resemblances, high-grade MFS is known as the myxoid variant of malignant fibrous histiocytoma [10]. MFS has been reported to have significantly higher recurrence rates, more than other soft tissue sarcomas do, ranging from $32 \%$ to $60 \%[9,11,12]$. Mentzel et al. [9] reported that $55.0 \%$ of MFS cases had local recurrences and $21.7 \%$ developed metastases. They also demonstrated that the local recurrence rate was independent of histological grade and tumor depth. DNA aneuploidy is associated with histological grade but not with the clinical course.

LMS is a malignant cancer of smooth muscle. LMS is a relatively rare tumor, accounting for $5 \%$ to $10 \%$ of all soft tissue sarcomas [13]. LMS occurs mostly in the retroperitoneum and other intra-abdominal lesions, including the mesentery. It is very difficult to accurately predict the clinical behavior of LMS. LMS can remain dormant for long periods and recur many years later. Massi et al. [14] provided evidence that LMS has an aggressive biologic activity. Early surgery, with margins of removal that are as wide as possible, is the most effective intervention [15]. Advanced age, vascular invasion, DNA aneuploidy, high mitotic rate, large tumor size, and 
American Joint Committee on Cancer (AJCC) staging may have prognostic value $[13,14,16]$.

Here, we report a case of high-grade extremity MFS with a synchronous mesenteric LMS. The synchronous development of multiple primary sarcomas of different histopathology is extremely rare [17] and very few cases have been reported in the literature $[4,18,19]$. In their review of 5505 sarcomas, Grobmyer et al. [20] reported only for 2 patients $(0.04 \%)$ who presented with synchronous soft tissue sarcomas of distinct pathology. This was also the case for sarcomas associated with other malignnant neoplasms, such as lymphoma [21,22] or adenocarcinoma $[23,24]$. Both Merimsky et al. [25] and Geva et al. [26] have respectively shown that $7.5 \%$ of soft tissue sarcoma patients developed other malignant neoplasms either before or after the initial sarcoma diagnosis. They presented that the incidence of second primary sarcomas in patients who were diagnosed previously with soft tissue sarcoma was 7.5 to 12.5 times greater than the incidence of primary soft tissue sarcoma in the general population $[20,25]$.

How multiple primary tumors occur in the same individual is unclear. Several explanations have been proposed. One of the greatest contributing factors is genetic susceptibility. Fon et al. [27] provided a novel hypothesis that common genetic factors, such as germ-line P53 mutations or mutations in checkpoint kinase-2, predispose patients to developing multiple synchronous tumors. Using array-based comparative genomic hybridization techniques, Wa et al. [28] demonstrated that LMS was characterized by frequent loss of 13q, loss of $10 \mathrm{q}$ and gain of 17p. Hernando et al. [29] found that mice carrying homozygous deletion of Pten alleles developed widespread smooth muscle cell hyperplasia and abdominal LMS. Little is known about the genetic association between MFS and LMS. Congyang et al. [21] proposed that histiocytic sarcoma have shared common clonal origins with diffuse large B cell lymphoma, or transdifferentiation theory. Other possible mechanisms included alkylating agents provoking chromosomal translocations of normal mesenchymal tissue [19], radiation [30] and persistent dysregulation of the immune system [31].

High-grade, advanced or malignant soft tissue sarcomas are aggressive diseases with poor prognosis and are usually invasive and metastasize [17]. Complete en bloc surgical resection is the single most important factor for better prognosis. Our case highlights the importance of thorough surgical and pathologic examination for all masses in a patient with synchronously detected sarcomas. Assuming that a mass is detected by radiological imaging in a patient with malignancy, the lesion might be interpreted as metastasis when a specific primary malignancy has a great tendency for distant metastasis. In our case, the mesenteric mass was initially misdiagnosed as metastasis of the coexistent primary tumor, thus precluding its staging. However, our histological result showed two obviously different synchronous sarcomas. In conclusion, both metastasis and multiple primary tumors should always be taken into consideration in the differential diagnosis when synchronous sarcomas are encountered. Recently, Park et al. [32] emphasized performing adequate pre-surgical evaluation and a comprehensive biopsy, even though a painless, movable soft tissue sarcoma of the extremity is likely a benign tumor. Takemoto et al. [24] emphasized the necessity of using immunohistochemistry in the differential diagnosis of sarcomas.

\section{Acknowledgements}

All authors of this manuscript have no financial conflicts of interest to disclose.

\section{REFERENCES}

[1] E. G. Demicco and A. J. Lazar, "Clinicopathologic Considerations: How Can We Fine Tune Our Approach to Sarcoma?" Seminars in Oncology, Vol. 38, Suppl. 3, 2011, pp. S3-18. doi:10.1053/j.seminoncol.2011.09.001

[2] D. G. Hope and J. J. Mulvihill, "Malignancy in Neurofibromatosis," Advances in Neurology, Vol. 29, 1981, pp. 33-56.

[3] D. H. Abramson, M. R. Melson, I. J. Dunkel and C. M. Frank, "Third (Fourth and Fifth) Nonocular Tumors in Survivors of Retinoblastoma," Ophthalmology, Vol. 108, No. 10, 2001, pp. 1868-1876. doi:10.1016/S0161-6420(01)00713-8

[4] P. Aurello, C. Cicchini, R. De Angelis, et al., "Synchronous and Metachronous Retroperitoneal Sarcomas: Two Case Reports," Anticancer Research, Vol. 22, No. 4, 2002, pp. 2409-2412.

[5] F. P. Li, J. F. Fraumeni, J. J. Mulvihill, et al., "A Cancer Family Syndrome in Twenty-Four Kindreds," Cancer Research, Vol. 48, No. 18, 1988, pp. 5358-5362.

[6] S. Sleijfer, C. Seynaeve and J. Verweij, "Using Single-Agent Therapy in Adult Patients with Advanced Soft Tissue Sarcoma Can Still Be Considered Standard Care," Oncologist, Vol. 10, No. 10, 2005, pp. 833-841.

[7] M. Tascilar, W. J. Loos, C. Seynaeve, J. Verweij and S. Sleijfer, "The Pharmacologic Basis of Ifosfamide Use in Adult Patients with Advanced Soft Tissue Sarcomas," Oncologist, Vol. 12, No. 11, 2007, pp. 1351-1360.

[8] R. W. Mutter, S. Singer, Z. Zhang, M. F. Brennan and K. M. Alektiar, "The Enigma of Myxofibrosarcoma of the Extremity," Cancer, Vol. 118, No. 2, 2012, pp. 518-527. doi:10.1002/cncr.26296

[9] T. Mentzel, E. Calonje, C. Wadden, et al., "Myxofibrosarcoma. Clinicopathologic Analysis of 75 Cases with Emphasis on the Low-Grade Variant," The American Journal of Surgical Pathology, Vol. 20, No. 4, 1996, pp. 391-405. 
doi:10.1097/00000478-199604000-00001

[10] S. W. Weiss and F. M. Enzinger, "Myxoid Variant of Malignant Fibrous Histiocytoma," Cancer, Vol. 39, No. 4, 1977, pp. 1672-1685.

doi:10.1002/1097-0142(197704)39:4<1672::AID-CNCR2 820390442>3.0.CO;2-C

[11] L. Angervall, L. G. Kindblom and C. Merck, "Myxofibrosarcoma. A Study of 30 Cases," Acta Pathologica Microbiologica Scandinavica Section A Pathology, Vol. 85A, No. 2, 1977, pp. 127-140. doi:10.1111/j.1699-0463.1977.tb00410.x

[12] A. Gronchi, S. Lo Vullo, C. Colombo, et al., "Extremity Soft Tissue Sarcoma in a Series of Patients TREATED at a Single Institution: Local Control Directly Impacts Survival," Annals of Surgery, Vol. 251, No. 3, 2010, pp. 506-511. doi:10.1097/SLA.0b013e3181cf87fa

[13] P. Gustafson, H. Willen, B. Baldetorp, et al., "Soft Tissue Leiomyosarcoma. A Population-Based Epidemiologic and Prognostic Study of 48 Patients, Including Cellular DNA Content," Cancer, Vol. 70, No. 1, 1992, pp. 114-119. doi:10.1002/1097-0142(19920701)70:1<114::AID-CNCR $\underline{2820700119>3.0 . C O ; 2-U}$

[14] D. Massi, G. Beltrami, M. M. Mela, et al., "Prognostic Factors in Soft Tissue Leiomyosarcoma of the Extremities: A Retrospective Analysis of 42 Cases," European Journal of Surgical Oncology, Vol. 30, No. 5, 2004, pp. 565-572. doi:10.1016/j.ejso.2004.03.002

[15] G. D. Demetri, S. Antonia, R. S. Benjamin, et al., "Soft Tissue Sarcoma," Journal of the National Comprehensive Cancer Network, Vol. 8, No. 6, 2010, pp. 630-674.

[16] K. Miyajima, Y. Oda, Y. Oshiro, et al., "Clinicopathological Prognostic Factors in Soft Tissue Leiomyosarcoma: A Multivariate Analysis," Histopathology, Vol. 40, No. 4, 2002, pp. 353-359. doi:10.1046/j.1365-2559.2002.01361.x

[17] A. Tsang, J. R. Nash, M. V. Fordham, M. N. Hartley and G. J. Poston, "The Management of Retroperitoneal Liposarcoma with Synchronous Intra-Duodenal Sarcoma," European Journal of Surgical Oncology, Vol. 29, No. 6, 2003, pp. 515-518. doi:10.1016/S0748-7983(03)00050-7

[18] A. Hadidy, A. Alsharif, R. Sheikh-Ali, et al., "Odontogenic Myxofibroma Synchronous with Primary Angiosarcoma of the Spleen," British Journal of Radiology, Vol. 83, No. 985, 2010, pp. e10-13. doi:10.1259/bjr/14078580

[19] K. Tsutsumi, Y. Aida, T. Ohno and M. Ookura, "Primary cardiac Rhabdomyosarcoma Following a Uterine Leiomyosarcoma: Double Primary Sarcomas," Japanese Journal of Thoracic and Cardiovascular Surgery, Vol. 53, No. 8, 2005, pp. 458-462. doi:10.1007/s11748-005-0086-7

[20] S. R. Grobmyer, N. Luther, C. R. Antonescu, S. Singer and M. F. Brennan, "Multiple Primary Soft Tissue Sarcomas," Cancer, Vol. 101, No. 11, 2004, pp. 2633-2635. doi:10.1002/cncr.20679

[21] L. Congyang, W. Xinggui, L. Hao and H. Weihua, "Synchronous Histiocytic Sarcoma and Diffuse Large B Cell Lymphoma Involving the Stomach: A Case Report and
Review of the Literature," International Journal of Hematology, Vol. 93, No. 2, 2011, pp. 247-252. doi:10.1007/s12185-011-0773-3

[22] A. Cossu, A. Deiana, A. Lissia, et al., "Synchronous Interdigitating Dendritic Cell Sarcoma and B-Cell Small Lymphocytic Lymphoma in a Lymph Node," Archives of Pathology \& Laboratory Medicine, Vol. 130, No. 4, 2006, pp. 544-547.

[23] M. Rettenmaier, H. D. Epstein, L. N. Abaid, K. A. Bechtol and B. H. Goldstein, "Leiomyosarcoma with Synchronous Clear Cell Ovarian Carcinoma," Onkologie, Vol. 33, No. 12, 2010, pp. 695-697. doi:10.1159/000322216

[24] Y. Takemoto, M. Kawahara, S. Yamamoto, et al., "Synchronous Primary Adenocarcinoma of the Lung and Leiomyosarcoma of the Small Intestine," Internal Medicine, Vol. 39, No. 8, 2000, pp. 655-658. doi:10.2169/internalmedicine.39.655

[25] O. Merimsky, Y. Kollender, J. Issakov, et al., "Multiple Primary Malignancies in Association with Soft Tissue Sarcomas," Cancer, Vol. 91, No. 7, 2001, pp. 1363-1371. doi:10.1002/1097-0142(20010401)91:7<1363::AID-CNC $\underline{\mathrm{R} 1140>3.0 . \mathrm{CO} ; 2-\mathrm{F}}$

[26] R. Geva, I. Jiveliouk, M. Inbar, et al., "The Co-Occurrence of Breast Cancer and Soft Tissue Sarcoma in a Single Cohort Series," American Journal of Clinical Oncology, Vol. 32, No. 1, 2009, pp. 34-37. doi:10.1097/COC.0b013e31817b6087

[27] A. Fon, L. Mileshkin, J. Slavin, et al., "Synchronous Diagnosis of Hodgkin Lymphoma and Osteosarcoma," Internal Medicine Journal, Vol. 37, No. 10, 2007, pp. 731732. doi:10.1111/j.1445-5994.2007.01465.X

[28] C. V. Wa, S. DeVries, Y. Y. Chen, F. M. Waldman and E. S. Hwang, "Clinical Application of Array-Based Comparative Genomic Hybridization to Define the Relationship between Multiple Synchronous Tumors," Modern Pathology, Vol. 18, No. 4, 2005, pp. 591-597. doi:10.1038/modpathol.3800332

[29] E. Hernando, E. Charytonowicz, M. E. Dudas, et al., "The AKT-mTOR Pathway Plays a Critical Role in the Development of Leiomyosarcomas," Nature Medicine, Vol. 13, No. 6, 2007, pp. 748-753. doi:10.1038/nm1560

[30] V. de Giorgi, R. Santi, M. Grazzini, et al., "Synchronous Angiosarcoma, Melanoma and Morphea of the Breast Skin 14 Years after Radiotherapy for Mammary Carcinoma," Acta Dermato-Venereologica, Vol. 90, No. 3, 2010, pp. 283-286.

[31] N. Ally, D. Kumar and F. M. Wodajo, "Synchronous Fibrosarcoma and Medullary Thyroid Cancer in a Man with AIDS," American Journal of Clinical Oncology, Vol. 29, No. 5, 2006, pp. 532-533. doi:10.1097/01.coc.0000177913.82468.ba

[32] J. H. Park, C. H. Kang, C. H. Kim and I. J. Chae, "Highly Malignant Soft Tissue Sarcoma of the Extremity with a Delayed Diagnosis," World Journal of Surgical Oncology, Vol. 8, 2010, p. 84. doi:10.1186/1477-7819-8-84 